\title{
Peripheral Fundus Findings in X-Linked Retinoschisis
}

\author{
Abigail T. Fahim ${ }^{1}$, MD, PhD, Naser Ali², MB, BChir, Taylor Blachley ${ }^{1}$, MS, \\ Michel Michaelides ${ }^{2,3}, \mathrm{MD}(\mathrm{Res}), \mathrm{FRCOphth}$ \\ ${ }^{1}$ Kellogg Eye Center, University of Michigan, Ann Arbor, MI, United States of America. \\ ${ }^{2}$ Moorfields Eye Hospital, 162 City Road, EC1V 2PD London, United Kingdom. \\ ${ }^{3}$ University College London, Institute of Ophthalmology, 11-43 Bath Street, EC1V 9EL \\ London, United Kingdom. \\ Corresponding Author: \\ Michel Michaelides \\ UCL Institute of Ophthalmology \\ 11-43 Bath Street, EC1V 9EL \\ London, United Kingdom \\ Email michel.michaelides@ucl.ac.uk \\ Phone +4402076086864
}

Word Count: 2,613 


\section{SYNOPSIS:}

In a large cohort of patient with X-linked retinoschisis, peripheral schisis was associated with vitreous hemorrhage and retinal detachment. Each additional peripheral fundus abnormality increased the odds of retinal detachment by a factor of 4.06 .

\section{ABSTRACT}

Background/Aims: Vitreous hemorrhage (VH) and retinal detachment (RD) cause a precipitous decline in vision in a subset of patients with X-linked retinoschisis (XLRS), an otherwise a slowly progressive condition. This study aims to report the frequency of macular and peripheral retinal findings in a large cohort of X-linked retinoschisis (XLRS) patients and to determine whether peripheral retinal findings are associated with $\mathrm{VH}$ and RD.

Methods: A retrospective observational case series was performed with 65 patients with XLRS with a pathogenic variant in RS1. Chart review included examination notes, fundus photographs, and optical coherence tomography (OCT). Fisher exact tests and univariable logistic regression analysis were used to determine the association between peripheral retinal findings (including retinoschisis, metallic sheen, vascular sheathing, pigmentary changes, white spiculations, and vitreous veils) and complications (including $\mathrm{VH}$ and $\mathrm{RD}$ ).

Results: Seven eyes (8\%) showed normal macular structure on OCT. Peripheral retinoschisis was significantly associated with both $\mathrm{VH}$ and RD. Out of 10 eyes with complications, $9(90 \%)$ had peripheral retinoschisis, compared with 33 out of 116 eyes (28\%) without complications ( $p=0.0014)$. In addition, each additional peripheral finding increased the odds of RD by a factor of $4.06(95 \% \mathrm{Cl} 1.58-10.39, \mathrm{p}=0.028)$. There were no complications in the 28 eyes with a normal periphery $(\mathrm{p}=0.84)$ or in the 35 eyes with metallic sheen $(p=0.42)$.

Conclusion: The data suggest that patients with peripheral retinoschisis are at increased risk for $\mathrm{VH}$ and $\mathrm{RD}$. Furthermore, patients with additional peripheral retinal findings together with peripheral schisis may carry additional risk for RD. 


\section{INTRODUCTION}

X-linked retinoschisis (XLRS) is an inherited retinal degeneration presenting in the first decade of life and characterized by a splitting of the inner retina in the macula with cystic cavities. Approximately $50 \%$ of individuals have schisis cavities in the peripheral retina as well ${ }^{1}$. Other peripheral retinal findings may include metallic sheen, retinal pigment epithelium (RPE) pigmentary changes, white spiculations, vascular sheathing, and vitreous veils ${ }^{1}$. The natural history is variable, with some patients experiencing deterioration in the first and second decade (20/60 to 20/120), whilst others remain relatively stable in childhood; further deterioration can occur later in adulthood secondary to outer retinal atrophy ${ }^{2}$. Patients are at increased risk for vitreous hemorrhage and retinal detachment, and these two complications primarily account for severe visual deterioration at a younger age in affected patients. Retinal detachments are typically rhegmatogenous, caused by breaks in the inner and outer walls of the schisis cavity, but tractional detachments can also occur. Vitreous hemorrhage is thought to occur in association with retinal breaks and vitreoretinal traction.

XLRS is caused by mutation in retinoschisin 1 (RS1), which has a discoidin domain and is implicated in cell-cell adhesion, consistent with the phenotype of schisis in the setting of $R S 1$ mutations $^{3}$. RS1 is expressed in photoreceptors, but the protein is present in both the outer and inner retina ${ }^{4}$.

Given the potentially devastating visual consequences of vitreous hemorrhage and retinal detachment, a clinically relevant question is whether peripheral retinal findings are associated with these complications. It is biologically plausible that peripheral schisis would predispose patients, and other peripheral retinal changes may play a role as well. Others have noted peripheral schisis in patients with vitreous hemorrhage ${ }^{5}$ and retinal detachment ${ }^{6}$, although prior reports lack statistical analysis. We hypothesize that peripheral schisis and other peripheral retinal changes are associated with an increased risk of vitreous hemorrhage and retinal detachment in XLRS patients. This question was addressed with a retrospective chart review of 65 patients with molecularly-proven XLRS.

\section{METHODS}

\section{Patients}

This was a retrospective review of medical records of all patients seen at Moorfields Eye Hospital with XLRS, diagnosed based on clinical findings and family history, and confirmed by detection of a disease-causing variant in RS1. Patients without fundus photos on record were excluded. In total 65 patients were ascertained, with visit dates between 1965 and 2015, and follow-up ranging from a single encounter to 48 years. Although some patients had visit dates as far back as 1965, all patients had visit dates recent enough to have genetic testing. Ethical approval was obtained from Moorfields Eye Hospital for this retrospective observational series.

\section{Clinical Evaluation}


All patients had undergone a comprehensive ophthalmologic examination, including measurement of best-corrected visual acuity, intraocular pressure, slit-lamp examination, dilated fundus examination, color fundus photography, and spectraldomain optical coherence tomography (SD-OCT). In addition, a proportion of patients had measurement of color vision, kinetic visual field, and electrophysiology.

Color fundus photography was performed with a TRC-50IA retinal fundus camera (Topcon, Tokyo, Japan) or an Optos ultra widefield camera (Optos plc, Scotland, UK). Spectral domain OCT was performed with the Spectralis OCT (Heidelberg Engineering, Heidelberg, Germany). Electrophysiology included full-field electroretinogram (ffERG) and pattern ERG ( $p E R G$ ), recorded with gold foil electrodes. Protocols incorporated the recommendations of the International Society for Clinical Electrophysiology of Vision ${ }^{78}$.

The main outcome measures were the frequency of peripheral retinal findings, including retinoschisis, metallic sheen, vascular sheathing, pigmentary changes, white spiculations, and vitreous veils; and frequency of complications, including vitreous hemorrhage and retinal detachment.

\section{Statistics}

Fisher exact tests were performed on the $2 \times 2$ cross-tabulations of peripheral retinal findings and complications, with a correction for multiple testing for 7 repeat tests (one for each peripheral finding, including a "normal" periphery). P-values were adjusted based on the multiple-testing correction. Univariable logistic regressions were performed to test the effect of multiple peripheral findings on odds of complications. Fisher exact tests were performed on the cross-tabulations of genotype and peripheral retinal findings, with a correction for multiple testing for 210 repeat tests (35 genotypes $x$ 6 peripheral retinal findings). P-values were adjusted based on the multiple-testing correction.

\section{RESULTS}

\section{Patients and Clinical Findings}

Sixty-five patients with molecularly-proven X-linked retinoschisis seen between 1965 and 2015 were included. They ranged in age from 3 to 65 years. Best-corrected visual acuity ranged from 6/9 to no perception of light. Color vision was documented in only 4 patients, ranging from 0/17 color plates to $15 / 17$. Full-field ERGs, documented in 16 patients, ranged from normal (one patient) to rod or rod-cone abnormalities with an electronegative waveform. Pattern ERGs, documented in 8 patients, were variably reduced.

\section{RS1 Sequence Variants}

Thirty-six different $R S 1$ likely disease-causing variants were identified, including 2 large deletions, 3 single nucleotide deletion/duplications, 3 splice site mutations, 1 nonsense mutation, and 27 missense mutations. Thirty mutations were previously reported ( 29 in the literature and 1 in the Leiden Open Variation Database) $)^{9-19}$ (Table 1). 
Table 1. Mutations in XLRS Cohort

\begin{tabular}{|c|c|c|}
\hline Mutation & \# pts & Previous Report \\
\hline Deletion of exon 1 & 1 & Retinoschisis Consortium ${ }^{7}$ \\
\hline c.35T>A (p.Leu12His) & 2 & Retinoschisis Consortium $^{7}$ \\
\hline c. $52+2 T>C$ & 1 & Khan et al. ${ }^{8}$ \\
\hline Deletion of exons 2 and 3 & 1 & Khan et al. ${ }^{8}$ \\
\hline c. 120 C>A (p.Cys40X) & 1 & Retinoschisis Consortium ${ }^{7}$ \\
\hline c. $185-1 G>A$ & 1 & Vincent et al. ${ }^{9}$ \\
\hline c. $184+2 T>G$ & 1 & Vincent et al. ${ }^{9}$ \\
\hline c.214G>A (p.Glu72Lys) & 3 & Retinoschisis Consortium ${ }^{7}$ \\
\hline c. $214 G>C$ (p.Glu72Gln) & 2 & Yu et al. ${ }^{10}$ \\
\hline c.239A >C (p.GIn80Pro) & 1 & Novel \\
\hline c.242T>A (p.lle81Asn) & 1 & Simonelli et al. ${ }^{11}$ \\
\hline c.276G>C (p.Trp92Cys) & 1 & Hotta et al. ${ }^{12}$ \\
\hline c.304C>T (p.Arg102Trp) & 6 & Retinoschisis Consortium ${ }^{7}$ \\
\hline c.305G>A (p.Arg102GIn) & 5 & Retinoschisis Consortium ${ }^{7}$ \\
\hline c.317A>C (p.GIn106Pro) & 1 & Novel \\
\hline c.325G>C (G109R) & 1 & Sauer et al. ${ }^{13}$ \\
\hline c.325G>T (p.G109W) & 1 & Sergeev et al. ${ }^{14}$ \\
\hline c.326G>C (p.Gly109Ala) & 1 & LOVD $^{17}$ \\
\hline c.329G>A (p.Cys110Tyr) & 2 & Retinoschisis Consortium ${ }^{7}$ \\
\hline c.337C>T (p.Leu113Phe) & 1 & Retinoschisis Consortium $^{7}$ \\
\hline c.378delT (p.Leu127Ter) & 1 & Novel \\
\hline c.421C>T (p.R141C) & 3 & Retinoschisis Consortium $^{7}$ \\
\hline c.435dupT (p.Glu146fs) & 1 & Novel \\
\hline c.496T>C (p.Tyr166His) & 1 & Chen et al. ${ }^{15}$ \\
\hline c.508A>C (p.Thr170Pro) & 1 & Novel \\
\hline c.526T>C (p.Phe176Val) & 1 & Novel \\
\hline c.554C>A (p.Thr185Lys) & 2 & Sergeev et al. ${ }^{14}$ \\
\hline c.574C>T (p.Pro192Ser) & 5 & Retinoschisis Consortium ${ }^{7}$ \\
\hline c.575C>T (p.Pro192Leu) & 1 & Lesch et al. ${ }^{16}$ \\
\hline c.579dupC (p.Ile194fs) & 3 & Retinoschisis Consortium ${ }^{7}$ \\
\hline c.589C>T (p.Arg197Cys) & 1 & Retinoschisis Consortium ${ }^{7}$ \\
\hline c.596T>C (p.lle199Thr) & 1 & Retinoschisis Consortium ${ }^{7}$ \\
\hline c.598C>T (p.Arg200Cys) & 4 & Retinoschisis Consortium $^{7}$ \\
\hline c.599G>A (p.Arg200His) & 1 & Retinoschisis Consortium ${ }^{7}$ \\
\hline c.608C>T (p.P203L) & 1 & Retinoschisis Consortium ${ }^{7}$ \\
\hline c.637C>T (p.Arg213Trp) & 2 & Retinoschisis Consortium ${ }^{7}$ \\
\hline
\end{tabular}

All variant positions are specified with respect to Ensembl transcript ENST0000037998422. \# pts= number of patients.
Six mutations were novel. None of the novel mutations are found in the Exome Aggregation Consortium ${ }^{20}$, and all are predicted "probably damaging" using Polyphen-2 21 .

\section{Macular Phenotype}

Ninety eyes in 47 patients had macular SD-OCT. Sixty-eight eyes $(76 \%)$ showed macular schisis on SD-OCT, ranging in age from 5 to 36 . Thirtyfive eyes (39\%) showed outer retinal atrophy, ranging in age from 29 to 64 . Twenty eyes $(22 \%)$ showed both macular schisis and outer retinal atrophy, ranging in age from 33 to 64 . Seven eyes (8\%) in 4 patients showed no schisis or atrophy, ranging in age from 11 to 45 . The 45 yearold was an outlier, with the other patients ranging from 11 to 20 years of age. All 7 eyes with a normal macula had peripheral retinal abnormalities. Four eyes had pigmentary changes, three eyes had peripheral schisis, 2

eyes had white spiculations, and 2 eyes had metallic sheen. All 8 patients had missense mutations in $R S 1$. Out of the 43 patients that had SD-OCT in both eyes, 40 patients $(93 \%)$ had concordant phenotypes in the two eyes, while 3 patients $(7 \%)$ had discordant phenotypes.

\section{Peripheral Retinal Phenotype}

There were 126 eyes with fundus photos. Twenty-eight (24\%) had a normal peripheral retina. Peripheral retinal findings, from most to least common, included retinoschisis in 
42 eyes (33\%), metallic sheen in 35 eyes (28\%), vitreous veils in 24 eyes (19\%), RPE pigment migration in 20 eyes (16\%), white spiculations in 11 eyes (9\%), and vascular sheathing in 8 eyes $(6 \%)$ (Table 2). Representative fundus photos of relevant peripheral retinal findings are shown in Figure 1.

Table 2. Frequency of peripheral retinal findings and complications of vitreous hemorrhage and retinal detachment in XLRS cohort

\begin{tabular}{cccccccc}
\hline & All Eyes & VH or RD & VH & RD & RRD & TRD & ExRD \\
\hline Total & 126 & $10(7.9)$ & $4(3.2)$ & $8(6.3)$ & 4 & 3 & 1 \\
Normal Periphery & $28(24.1)$ & $0(0.0)$ & $0(0.0)$ & $0(0.0)$ & $0(0.0)$ & $0(0.0)$ & $0(0.0)$ \\
Vitreous Veils & $24(19.0)$ & $2(20.0)$ & $0(0.0)$ & $2(25.0)$ & $1(25.0)$ & $1(33.3)$ & $0(0.0)$ \\
Sheen & $35(27.8)$ & $0(0.0)$ & $0(0.0)$ & $0(0.0)$ & $0(0.0)$ & $0(0.0)$ & $0(0.0)$ \\
Schisis & $42(33.3)$ & $9(90.0)$ & $4(100.0)$ & $7(87.5)$ & $4(100.0)$ & $2(66.7)$ & $1(100.0)$ \\
White Spiculations & $11(8.7)$ & $2(20.0)$ & $0(0.0)$ & $2(25.0)$ & $2(50.0)$ & $0(0.0)$ & $0(0.0)$ \\
Vascular Sheathing & $8(6.3)$ & $1(10.0)$ & $0(0.0)$ & $1(12.5)$ & $0(0.0)$ & $0(0.0)$ & $1(100.0)$ \\
Pigment & $20(15.9)$ & $4(40.0)$ & $1(25.0)$ & $4(50.0)$ & $2(50.0)$ & $1(33.3)$ & $1(100.0)$ \\
\hline
\end{tabular}

$\mathrm{VH}=$ vitreous hemorrhage. $\mathrm{RD}=$ retinal detachment. $\mathrm{RRD}=$ rhegmatogenous retinal detachment. $\mathrm{TRD}=$ tractional retinal detachment. ExRD= exudative retinal detachment. Values are shown as number of eyes (with percentage of the total in parenthesis).

\section{Genotype-Phenotype Correlations}

All peripheral findings were compared across genotypes to define genotype-phenotype correlations. All 6 eyes in all 3 patients with the c.214G>A(p.Glu72Lys) mutation had vitreous veils, which was statistically significant $(p=0.021)$. The 2 most common mutations, c.304C>T(Arg102Trp) and c.305G>A(Arg102Gln), showed 8 out of 12 eyes with peripheral metallic sheen and 3 out of 10 eyes with peripheral white spiculations, respectively. However, this did not reach statistical significance.

\section{Complications}

Occurrences of vitreous hemorrhages and retinal detachments were determined by documentation in the patient notes, and were corroborated by fundus photos when available. There were 4 eyes with vitreous hemorrhage (3\%) and 8 eyes with retinal detachment (6\%) (Table 2). There were no retinal detachments or vitreous hemorrhages in eyes with a normal peripheral retinal exam $(p=0.84)$, or in eyes with metallic sheen $(p=0.42)$ (Table 3).

Out of 10 eyes with complications, $9(90 \%)$ had peripheral retinoschisis, compared with only $28 \%$ of eyes without complications $(p=0.0014)$. This includes 4 out of 4 eyes with vitreous hemorrhage $(p=0.07)$ and 7 out of 8 eyes with retinal detachment $(p=0.014)$ that showed peripheral schisis (Table 3). In addition, the number of peripheral findings was significantly associated with the odds of retinal detachment, with each additional peripheral finding increasing a patient's odds of detachment by a factor of $4.06(95 \% \mathrm{Cl}$ 1.58-10.39, $p=0.028$ ). There was no similar effect on the odds of vitreous hemorrhage. 
Table 3. Peripheral retinoschisis is significantly associated with complications.

\begin{tabular}{|c|c|c|c|c|c|c|c|c|c|c|}
\hline & & \multicolumn{3}{|c|}{ Any Complication } & \multicolumn{3}{|c|}{ Vitreous Hemorrhage } & \multicolumn{3}{|c|}{ Retinal Detachment } \\
\hline & & No & Yes & $\begin{array}{c}p- \\
\text { value }\end{array}$ & No & Yes & $\begin{array}{c}p- \\
\text { value }\end{array}$ & No & Yes & $\begin{array}{c}\mathrm{p}- \\
\text { value }\end{array}$ \\
\hline & Total & 116 & 10 & & 122 & 4 & & 118 & 8 & \\
\hline \multirow{2}{*}{$\begin{array}{l}\text { Peripheral } \\
\text { Schisis }\end{array}$} & No & $\begin{array}{c}83 \\
(71.6)\end{array}$ & $1(10.0)$ & \multirow{2}{*}{0.0014} & $\begin{array}{c}84 \\
(68.9)\end{array}$ & $0(0.0)$ & \multirow{2}{*}{0.07} & $\begin{array}{c}83 \\
(70.3)\end{array}$ & $1(12.5)$ & \multirow{2}{*}{0.014} \\
\hline & Yes & $\begin{array}{c}33 \\
(28.4)\end{array}$ & $9(90.0)$ & & $\begin{array}{c}38 \\
(31.1)\end{array}$ & $\begin{array}{c}4 \\
(100.0)\end{array}$ & & $\begin{array}{c}35 \\
(29.7)\end{array}$ & $7(87.5)$ & \\
\hline \multirow{2}{*}{$\begin{array}{l}\text { Normal } \\
\text { Periphery }\end{array}$} & No & $\begin{array}{c}88 \\
(75.9)\end{array}$ & $\begin{array}{c}10 \\
(100.0)\end{array}$ & \multirow{2}{*}{0.84} & $\begin{array}{c}94 \\
(77.0)\end{array}$ & $\begin{array}{c}4 \\
(100.0)\end{array}$ & \multirow{2}{*}{1.00} & $\begin{array}{c}90 \\
(76.3)\end{array}$ & $\begin{array}{c}8 \\
(100.0)\end{array}$ & \multirow{2}{*}{1.00} \\
\hline & Yes & $\begin{array}{c}28 \\
(24.1)\end{array}$ & $\begin{array}{c}0 \\
(0.0)\end{array}$ & & $\begin{array}{c}28 \\
(23.0)\end{array}$ & $\begin{array}{c}0 \\
(0.0)\end{array}$ & & $\begin{array}{c}28 \\
(23.7)\end{array}$ & $\begin{array}{c}0 \\
(0.0)\end{array}$ & \\
\hline \multirow{2}{*}{$\begin{array}{l}\text { Peripheral } \\
\text { Sheen }\end{array}$} & No & $\begin{array}{c}81 \\
(69.8)\end{array}$ & $\begin{array}{c}10 \\
(100.0)\end{array}$ & \multirow{2}{*}{0.42} & $\begin{array}{c}87 \\
(71.3)\end{array}$ & $\begin{array}{c}4 \\
(100.0)\end{array}$ & \multirow{2}{*}{1.00} & $\begin{array}{c}83 \\
(70.3)\end{array}$ & $\begin{array}{c}8 \\
(100.0)\end{array}$ & \multirow{2}{*}{0.70} \\
\hline & Yes & $\begin{array}{c}35 \\
(30.2) \\
\end{array}$ & $\begin{array}{c}0 \\
(0.0) \\
\end{array}$ & & $\begin{array}{c}35 \\
(28.7) \\
\end{array}$ & $\begin{array}{c}0 \\
(0.0)\end{array}$ & & $\begin{array}{c}35 \\
(29.7)\end{array}$ & $\begin{array}{c}0 \\
(0.0)\end{array}$ & \\
\hline
\end{tabular}

Values are shown as number of eyes (with percentage of the total in parenthesis). Significant $p$-values are in bold.

\section{DISCUSSION}

The natural history of XLRS is highly variable, but if there is progression it is often very slow in childhood and early adulthood, potentially followed by additional gradual decline in later adulthood from macular atrophy. This chronic clinical course can be interrupted by a precipitous drop in vision due to complications of vitreous hemorrhage or retinal detachment, often at a young age. This study describes the frequency of these complications in a cohort of 65 patients from a single center with molecularly-proven $\mathrm{XLRS}$, as well as the frequency of peripheral retinal findings. The study is limited by the small sample size, the retrospective design, and the widely variable follow-up of subjects.

We report macular schisis in $76 \%$ of eyes in our cohort. Previous reports in the literature range from $68 \%$ to $100 \%{ }^{156} 23$. The age distribution in the cohort is expected to significantly impact the prevalence of macular schisis, since the natural history of disease is macular schisis followed by macular atrophy and resolution of schisis. Therefore, clinicians typically view macular atrophy as a presumptive sign of prior schisis. George et al. and Kellner et al. report macular schisis in $68 \%$ and $71 \%$ of eyes respectively, but both report that $100 \%$ of eyes showed macular pathology on examination, in the form of schisis, blunted fovea, pigment irregularities, or RPE atrophy ${ }^{16}$. In contrast, we report 7 eyes in 4 patients with normal macular structure on SD-OCT ( $8 \%$ of eyes). The previous reports, with the exception of Wang et al., were published before the RS1 gene was identified and the diagnosis was therefore based on 
clinical examination and family history, with family linkage to the XLRS locus in the case of George et al. In the current study the molecular diagnosis was confirmed in all patients, thus allowing identification of patients with a normal macula despite a pathologic $R S 1$ variant. However, this may not fully explain the discrepancy as all 7 eyes had peripheral retina abnormalities and perhaps would have been classified as XLRS even without genetic testing in the setting of a consistent family history. Previous investigations into genotype-phenotype correlations have found that patients with normal macular structure nearly always have missense mutations, although the inverse is not true: patients with those same missense mutations can have macular schisis and variable severity ${ }^{11}$. This is consistent with our data. All 4 patients with normal macular structure had missense mutations. In 3 out of 4 cases there were other patients with the same mutations that did show macular schisis. In the fourth case, there were discordant phenotypes between the 2 eyes: one eye had a normal macula with peripheral schisis, while the other eye had macular schisis, vitreous hemorrhage, and retinal detachment. Prior genotype-phenotype investigations have also revealed worse visual acuity and more frequent ERG abnormalities among patients with nonsense, splice-site, and frame-shift mutations compared to those with missense mutations ${ }^{11}$. It is well established that an electronegative bright flash dark-adapted ERG is a characteristic finding in XLRS, although it is not universal. The b-wave amplitude is variable and the a-wave amplitude can also be reduced, especially in those with significant peripheral retinoschisis or pigmentary changes ${ }^{11}$. Despite the large testretest variability of ERG recordings and the inherent limitations associated with ERG assessment in children (important given the majority of complications are in childhood), future prospective studies of bright flash dark adapted a- and b-wave amplitudes in relation to vitreous hemorrhage or retinal detachment may suggest these parameters as a functional marker for risk of complication.

We report $33 \%$ of eyes with peripheral retinoschisis, which is lower than previous reports, which have ranged from $43-60 \%{ }^{152324}$. Similar to macular schisis, the rate of peripheral schisis may also be impacted by the age distribution of the cohort, since schisis cavities sometimes reabsorb with time. The rates of other peripheral findings have been reported in George et al. (56 patients), in which reported rates were generally higher than the rates reported herein: metallic sheen in $38 \%$ of eyes (vs. $28 \%$ ), vitreous veils in $39 \%$ of eyes (vs. $19 \%$ ), pigmentary changes in $29 \%$ of eyes (vs. $16 \%$ ), white spiculations in $11 \%$ of eyes (vs. $9 \%$ ), and vascular sheathing in $9 \%$ of eyes (vs. 6\%) ${ }^{1}$. The complication rates reported here are also on the lower end of the range previously reported. We report vitreous hemorrhage in 3\% of eyes, compared to $4-21 \%$ in previous reports, and retinal detachment in $6 \%$ of eyes, compared to $5.5-16 \%$ in previous reports $^{15624}$.

We report here that the presence of peripheral retinoschisis is significantly associated with the complications of vitreous hemorrhage and retinal detachment, and furthermore that the odds of retinal detachment increase significantly with each additional peripheral retinal abnormality. In addition, there were no complications in eyes with a normal periphery or with metallic sheen, although this did not reach statistical significance. There was one patient with a complication (RD) and no peripheral schisis, although we 
cannot rule out that he had a previous schisis cavity that spontaneously reabsorbed. Kellner et al. propose that peripheral schisis is a risk factor for retinal detachment because peripheral schisis was present in $75 \%$ of fellow eyes in cases of retinal detachment, compared to $53 \%$ of all eyes without a history of retinal detachment ${ }^{6}$. More recently, Wang et al. reported vitreous hemorrhage in 4 out of 23 patients with XLRS, and all 4 had peripheral retinoschisis ${ }^{5}$. This study adds to the previous reports, which lack statistical analysis, by demonstrating a statistically significant association between peripheral schisis and complication rates, as well as an increasing likelihood of retinal detachment with each additional peripheral fundus finding. A clinically relevant question is whether these peripheral retinal findings precede the complications or appear subsequently as a result of the complications. Of note, these complications frequently happen in childhood and adolescence. Sometimes patients initially present with vitreous hemorrhage or retinal detachment, and no prior records exist. Kellner et al. report that all vitreous hemorrhages in their cohort occurred before the age of 10 , and most retinal detachments occurred before the age of 11 , with the exception of some longstanding detachments of unknown duration found later in life ${ }^{6}$. Patients in our cohort also presented with complications at a young age. Patients with vitreous hemorrhage ranged from 8 to 21 years old, and patients with retinal detachment ranged from 3 to 21 years old, plus one 28 year-old with an old retinal detachment of unknown duration. Unfortunately in most cases there were no records prior to the complication, with 2 exceptions. In one case a patient had an exudative retinal detachment at 21 years of age with previous documentation of vitreous veils in that eye at age 9 and peripheral schisis at age 14. Another patient had vitreous hemorrhage at age 11 and a tractional retinal detachment at age 12 with previously documented peripheral schisis and pigmentary changes in the same eye at age 5 . This demonstrates that at least in these cases the peripheral retinal findings preceded the complications. Furthermore, despite having numerous eyes with a normal periphery, we have no documented cases of a patient with normal periphery developing a subsequent complication. Finally, the presence of these peripheral retinal findings in large numbers of XLRS patients without complications argues that these findings are not a consequence of prior vitreous hemorrhage or retinal detachment. However, we cannot exclude that a patient may have had a vitreous hemorrhage that resolved without presenting to the ophthalmologist.

This study reports the frequency of both macular and peripheral retinal findings in a large cohort of molecularly proven XLRS patients. The data suggest that patients with peripheral retinoschisis are at increased risk for vitreous hemorrhage and retinal detachment. Furthermore, patients with additional peripheral retinal findings together with peripheral schisis may carry additional risk for retinal detachment. A normal retinal periphery and a retinal metallic sheen may both portend a good prognosis with respect to risk for complications. 


\section{ACKNOWLEDGEMENTS}

This work was supported by Moorfields Eye Hospital Special Trustees, grant number ST $1310 \mathrm{~F}$, Moofields Eye Charity, grant number MEC $1310 \mathrm{~A}$, and the Foundation Fighting Blindness, grant number CD-CL-0711-0518-UCL. Prof. Andrew Webster, MB, BChir, at Moorfields Eye Hospital in London, UK, managed many of the patients presented in this study and kindly gave us access to the data. He also provided feedback during manuscript preparation.

\section{COMPETING INTERESTS}

Abigail Fahim owns stock in lonis Pharmaceuticals (Carlsbad, CA). Michel Michaelides consults for Meira GTx (London, UK), Ora Inc (Andover, MA), Shire (Hampshire, UK), and Editas (Cambridge, MA). Naser Ali and Taylor Blachley have no financial disclosures.

\section{CONTRIBUTORSHIP}

The authors contributed in the following ways to this manuscript:

Abigail T Fahim participated in study design, data collection, manuscript preparation and editing. Naser Ali participated in data collection and manuscript editing.

Taylor Blachley participated in statistical analysis and manuscript editing. Michel Michaelides participated in study design, data collection, and manuscript editing. 


\section{REFERENCES}

1. George ND, Yates JR, Moore AT. Clinical features in affected males with X-linked retinoschisis. Arch Ophthalmol 1996;114(3):274-80.

2. Sieving PA, MacDonald IM, Chan S. X-Linked Juvenile Retinoschisis. In: Pagon RA, Adam MP, Ardinger HH, et al., eds. GeneReviews(R). Seattle (WA)1993.

3. Sergeev YV, Caruso RC, Meltzer MR, et al. Molecular modeling of retinoschisin with functional analysis of pathogenic mutations from human X-linked retinoschisis. Hum Mol Genet 2010;19(7):1302-13. doi: 10.1093/hmg/ddq006

4. Grayson C, Reid SN, Ellis JA, et al. Retinoschisin, the X-linked retinoschisis protein, is a secreted photoreceptor protein, and is expressed and released by Weri-Rb1 cells. Hum Mol Genet 2000;9(12):1873-9.

5. Wang NK, Liu L, Chen HM, et al. Clinical presentations of X-linked retinoschisis in Taiwanese patients confirmed with genetic sequencing. Mol Vis 2015;21:487-501.

6. Kellner U, Brummer S, Foerster MH, et al. X-linked congenital retinoschisis. Graefes Arch Clin Exp Ophthalmol 1990;228(5):432-7.

7. Marmor MF, Fulton AB, Holder GE, et al. ISCEV Standard for full-field clinical electroretinography (2008 update). Doc Ophthalmol 2009;118(1):69-77. doi: 10.1007/s10633-008-9155-4

8. Bach M, Brigell MG, Hawlina M, et al. ISCEV standard for clinical pattern electroretinography (PERG): 2012 update. Doc Ophthalmol 2013;126(1):1-7. doi: 10.1007/s10633-012-9353-y

9. Functional implications of the spectrum of mutations found in 234 cases with X-linked juvenile retinoschisis. The Retinoschisis Consortium. Hum Mol Genet 1998;7(7):1185-92.

10. Khan NW, Jamison JA, Kemp JA, et al. Analysis of photoreceptor function and inner retinal activity in juvenile X-linked retinoschisis. Vision Res 2001;41(28):3931-42.

11. Vincent A, Robson AG, Neveu MM, et al. A phenotype-genotype correlation study of Xlinked retinoschisis. Ophthalmology 2013;120(7):1454-64. doi: 10.1016/j.ophtha.2012.12.008

12. Yu P, Li J, Li R, et al. [Identification of mutation of the X-linked juvenile retinoschisis gene]. Zhonghua Yi Xue Yi Chuan Xue Za Zhi 2001;18(2):88-91.

13. Simonelli F, Cennamo G, Ziviello $C$, et al. Clinical features of X linked juvenile retinoschisis associated with new mutations in the XLRS1 gene in Italian families. $\mathrm{Br}$ J Ophthalmol 2003;87(9):1130-4.

14. Hotta Y, Fujiki K, Hayakawa M, et al. Japanese juvenile retinoschisis is caused by mutations of the XLRS1 gene. Hum Genet 1998;103(2):142-4.

15. Sauer CG, Gehrig A, Warneke-Wittstock R, et al. Positional cloning of the gene associated with X-linked juvenile retinoschisis. Nat Genet 1997;17(2):164-70. doi: 10.1038/ng1097-164

16. Sergeev YV, Vitale S, Sieving PA, et al. Molecular modeling indicates distinct classes of missense variants with mild and severe XLRS phenotypes. Hum Mol Genet 2013;22(23):4756-67. doi: 10.1093/hmg/ddt329

17. Chen J, Xu K, Zhang X, et al. Novel mutations of the RS1 gene in a cohort of Chinese families with X-linked retinoschisis. Mol Vis 2014;20:132-9. 
18. Lesch B, Szabo V, Kanya M, et al. Clinical and genetic findings in Hungarian patients with X-linked juvenile retinoschisis. Mol Vis 2008;14:2321-32.

19. Fokkema IF, Taschner PE, Schaafsma GC, et al. LOVD v.2.0: the next generation in gene variant databases. Hum Mutat 2011;32(5):557-63. doi: 10.1002/humu.21438

20. Exome Aggregation Consortium (ExAC). Cambridge, MA.

21. Adzhubei IA, Schmidt S, Peshkin L, et al. A method and server for predicting damaging missense mutations. Nat Methods 2010;7(4):248-9. doi: 10.1038/nmeth0410-248

22. Patel N, Aldahmesh MA, Alkuraya $\mathrm{H}$, et al. Expanding the clinical, allelic, and locus heterogeneity of retinal dystrophies. Genet Med 2016;18(6):554-62. doi: 10.1038/gim.2015.127 [published Online First: 2015/09/10]

23. Deutman A. Sex-linked juvenile retinoschisis. In: Deutman A, ed. The Hereditary Dystrophies of the Posterior Pole of the Eye. Assen, The Netherlands: Van Gorcum 1971:48-98.

24. Roesch MT, Ewing CC, Gibson AE, et al. The natural history of X-linked retinoschisis. Can J Ophthalmol 1998;33(3):149-58. 